\title{
Why should one normalize heart rate variability with respect to average heart rate
}

\author{
Jerzy Sacha* \\ Department of Cardiology, Regional Medical Center, Opole, Poland \\ *Correspondence: sacha@op.pl \\ Edited by: \\ Mikko Paavo Tulppo, Verve, Finland
}

Keywords: heart rate variability, heart rate, autonomic nervous system, analysis, R-R interval

Heart rate variability (HRV) is a recognized risk factor in many disease states (Bravi et al., 2011; Sacha et al., 2013a). However, HRV is significantly correlated with an average heart rate (HR), and this association is both physiologically and mathematically determined. The physiological determination comes from the autonomic nervous system activity (Task Force of the European Society of Cardiology, and the North American Society of Pacing and Electrophysiology, 1996), but the mathematical one is caused by the non-linear (inverse) relationship between R-R interval and HR (Sacha and Pluta, 2005a,b, 2008).

HRV may be estimated by using R-R interval (the most frequent method) or HR signals, yet, they both do not yield the same results since they are inversely related with each other-indeed, the analyses are mathematically biased (Sacha and Pluta, 2005a,b). If one uses R-R intervals, the same changes of HR cause much higher fluctuations of R-R intervals for the slow average HR than for the fast one (Figure 1A). Conversely, if one employs HR signals, the same changes of R-R intervals cause much higher fluctuations of HR for the fast than slow average HR (Figure 1B). Consequently, due to these mathematical reasons, HRV estimated from R-R intervals should negatively correlate with average HR (or positively with average R-R interval), but HRV estimated from HR signals should be positively correlated with average HR (or negatively with average R-R interval) (Sacha and Pluta, 2005a,b). Moreover, due to the inverse relationship between R-R interval and $\mathrm{HR}$, there is a possibility that a given patient may have higher HRV than another in terms of R-R intervals and lower HRV in terms of HRs-Figure 1C explains such a case (Sacha and Pluta, 2005a).

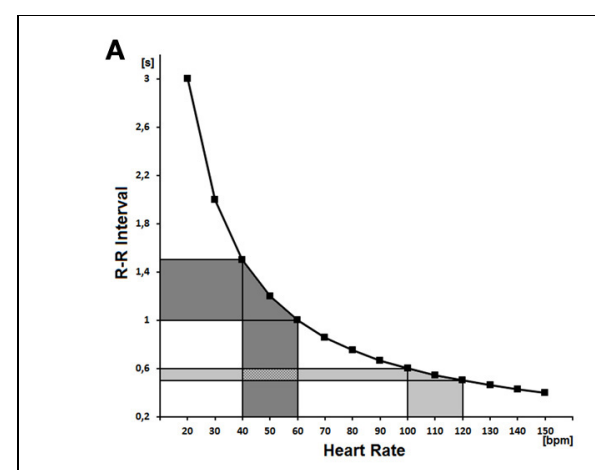

Another mathematical problem concerning the association between HRV and
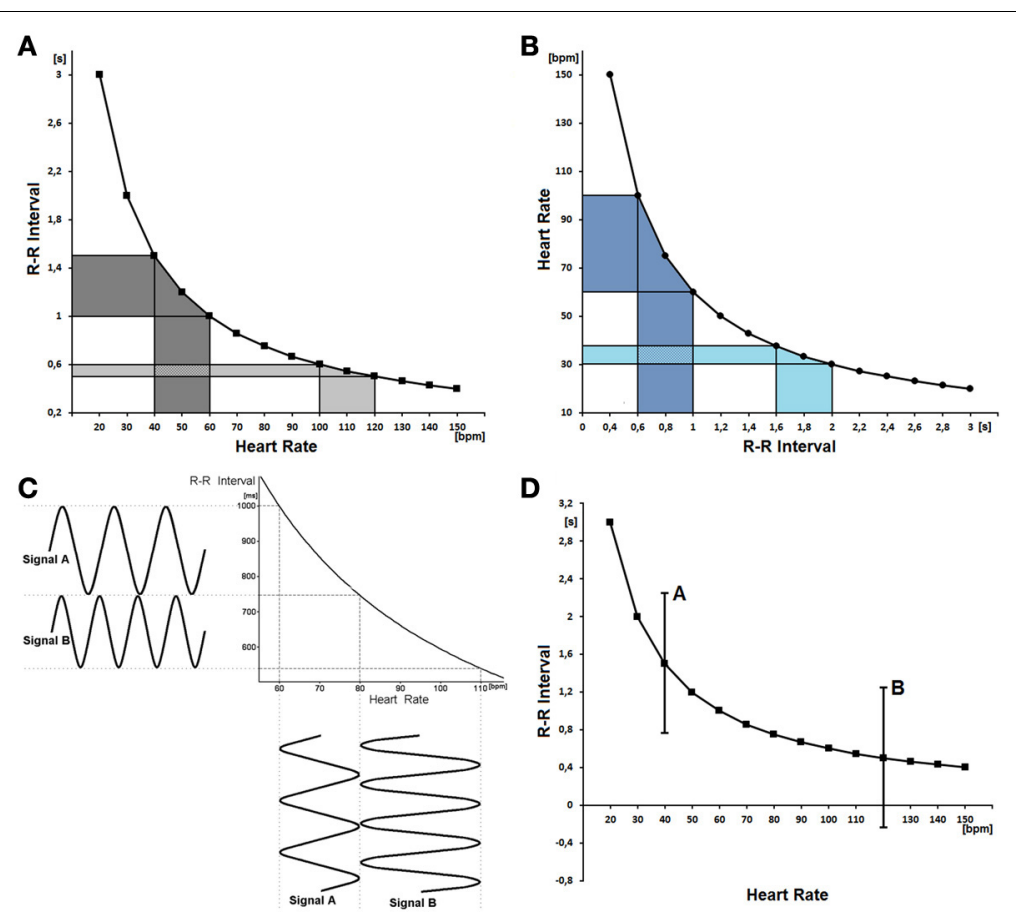

FIGURE 1 | (A) The non-linear (mathematical) relationship between R-R interval and heart rate is depicted. One can see that the oscillations of a slow average heart rate ( $x$-axis, dark gray area) result in much greater oscillations of RR intervals ( $y$-axis, dark gray area) than the same oscillations of a fast average heart rate (light gray area). As a consequence, the variability of $R-R$ intervals is higher for the slow average heart rate than for the fast one, despite the fact that the variability of heart rate is the same (reprinted with modification from Sacha and Pluta, 2008). (B) The relationship between heart rate and R-R interval is shown-the same oscillations of $R-R$ intervals yield much greater oscillations of $H R$ for the fast average heart rate (dark blue area) than for the slow one (light blue area). Consequently, the variability of $\mathrm{HR}$ is higher for the case with fast average heart rate, despite the fact that the variability of $R-R$ intervals is the same in both cases. (C) The relationship between R-R interval and heart rate is depicted along with two signals oscillating in different extents. Signal A oscillates between 60 and $80 \mathrm{bpm}$ but signal B between 80 and $110 \mathrm{bpm}$. One can see that signal A is more variable (its amplitude is higher) than signal $B$ when expressed as $R-R$ interval signals, and conversely signal $A$ is less variable than $B$ if expressed as HR ones. The example clearly shows how the same signals may reveal an inverse relationship with each other depending on the way they are expressed (reprinted from Sacha and Pluta, 2005a). (D) The relationship between $R-R$ interval and heart rate with two hypothetical examples of $R-R$ interval oscillations (i.e., $A$ and $B$ ) are presented. It is shown that the fluctuations of $R-R$ intervals may be potentially quite high for a slow average $H R(A)$, however, such fluctuations are not possible for a fast average HR (B) since the R-R intervals should have become negative. 
$\mathrm{HR}$ is presented in Figure 1D. One can see that the fluctuations of R-R intervals may be potentially very high for slow average HR, however, the same fluctuations are not possible for fast average $H R$, since the R-R intervals should have become negative. The same problem can be met if one calculates HRV from HR signals, i.e., the average HR of $80 \mathrm{bpm}$ may potentially fluctuate between 30 and $130 \mathrm{bpm}$ (i.e., the fluctuation amplitude equals $100 \mathrm{bpm}$ ), however, such fluctuations are not possible for the average HR of $40 \mathrm{bpm}$, since the heart rhythm must have fluctuated between -10 and $90 \mathrm{bpm}$.

Due to the above facts, the standard HRV analysis is mathematically biased, particularly if patients differ in terms of their average HRs. The only way to overcome it is to calculate HRV with respect to the average value, i.e., to normalize the fluctuations with respect to the mean (Sacha and Pluta, 2005a,b, 2008). One can do that by division of the signal by the average $\mathrm{R}-\mathrm{R}$ interval in the case of $\mathrm{R}-\mathrm{R}$ interval signal, or by the average $\mathrm{HR}$ in the case of HR signal. Moreover, this way the same results are obtained no matter if one calculates HRV from R-R intervals or HRs (Sacha and Pluta, 2005a).

Such an approach enables to explore the HR contribution to the physiological and clinical significance of HRV (Billman, 2013; Sacha et al., 2013a). Recently, this approach has been further developed to enhance or completely remove the
HR influence (even physiological one) on HRV, what turned out to provide valuable information on cardiac and non-cardiac prognosis in patients after myocardial infarction-the details have been published elsewhere (Sacha et al., 2013a,b,c).

To conclude, HRV is significantly associated with HR, which is caused by both physiological and mathematical phenomena, however, by a simple mathematical modification one may exclude mathematical bias and explore a real clinical value of HR and its variability.

\section{REFERENCES}

Billman, G. E. (2013). The effect of heart rate on the heart rate variability response to autonomic interventions. Front. Physiol. 4:222. doi: 10.3389/fphys.2013.00222

Bravi, A., Longtin, A., and Seely, A. J. (2011). Review and classification of variability analysis techniques with clinical applications. Biomed. Eng. Online 10, 90. doi: 10.1186/1475-925X-10-90

Sacha, J., and Pluta, W. (2005a). Which heart rate is more variable: a slow or a fast one?-It depends on the method of heart rate variability analysis. Folia Cardiol. 12(Suppl. D), 1-4.

Sacha, J., and Pluta, W. (2005b). Different methods of heart rate variability analysis reveal different correlations of heart rate variability spectrum with average heart rate. J. Electrocardiol. $38, \quad 47-53$. doi: 10.1016/j.jelectrocard. 2004.09.015

Sacha, J., and Pluta, W. (2008). Alterations of an average heart rate change heart rate variability due to mathematical reasons. Int. J. Cardiol. 128 , 444-447. doi: 10.1016/j.ijcard.2007.06.047

Sacha, J., Barabach, S., Statkiewicz-Barabach, G., Sacha, K., Müller, A., Piskorski, J., et al. (2013a). How to select patients who will not benefit from ICD therapy by using heart rate and its variability? Int. J. Cardiol. 168, 1655-1658. doi: 10.1016/j.ijcard. 2013.03.040

Sacha, J., Barabach, S., Statkiewicz-Barabach, G., Sacha, K., Muller, A., Piskorski, J., et al (2013b). How to strengthen or weaken the HRV dependence on heart rate-Description of the method and its perspectives. Int. J. Cardiol. 168, 1660-1663. doi: 10.1016/j.ijcard. 2013.03.038

Sacha, J., Sobon, J., Sacha, K., and Barabach, S. (2013c). Heart rate impact on the reproducibility of heart rate variability analysis. Int. J. Cardiol. doi: 10.1016/j.ijcard.2013.04.160. [Epub ahead of print].

Task Force of the European Society of Cardiology, and the North American Society of Pacing and Electrophysiology. (1996). Heart rate variability: standards of measurement, physiological interpretation and clinical use. Circulation 93, 1043-1065. doi: 10.1161/01.CIR.93.5.1043

Received: 18 September 2013; accepted: 04 October 2013; published online: 22 October 2013.

Citation: Sacha J (2013) Why should one normalize heart rate variability with respect to average heart rate. Front. Physiol. 4:306. doi: 10.3389/fphys.2013.00306 This article was submitted to Clinical and Translational Physiology, a section of the journal Frontiers in Physiology.

Copyright (C) 2013 Sacha. This is an open-access article distributed under the terms of the Creative Commons Attribution License (CC BY). The use, distribution or reproduction in other forums is permitted, provided the original author(s) or licensor are credited and that the original publication in this journal is cited, in accordance with accepted academic practice. No use, distribution or reproduction is permitted which does not comply with these terms. 